\title{
Effect of High-pressure Torsion on Corrosion Behavior of a Solution-treated Al-Mg-Sc Alloy in a Saline Solution
}

\author{
Michelle Dias Alves Lage $^{a}$, Renata Braga Soares ${ }^{a}{ }^{\mathbb{D}}$, Pedro Henrique R. Pereira $^{b, c}$, \\ Roberto B. Figueiredo ${ }^{c}$, Vanessa Freitas C. Lins ${ }^{a} *$, Terence G. Langdon ${ }^{b}$ \\ ${ }^{a}$ Departamento de Engenharia Química, Universidade Federal de Minas Gerais, \\ Belo Horizonte, MG, Brasil. \\ ${ }^{b}$ Materials Research Group, Department of Mechanical Engineering, University of Southampton, \\ Southampton SO17 1BJ, UK. \\ ${ }^{c}$ Departamento de Engenharia Metalúrgica e de Materiais, Universidade Federal de Minas Gerais, \\ Belo Horizonte, MG, Brasil.
}

Received: May 18, 2019; Revised: July 27, 2019; Accepted: September 18, 2019

The effect of the increase in crystalline defects in the structure generated by high-pressure torsion (HPT) on the corrosion behavior of metallic materials is not well understood. This report evaluates the influence of HPT on the corrosion behavior of a solution treated $\mathrm{Al}-3 \mathrm{wt} \% \mathrm{Mg}-0.2 \mathrm{wt} \% \mathrm{Sc}$ alloy in a 3.5 wt./v. $\% \mathrm{NaCl}$ solution. The electrochemical behavior of the alloy was evaluated using cyclic polarization, electrochemical impedance spectroscopy (EIS), Mott Schottky, X-ray photoelectron spectroscopy, and chronoamperometry testing. The passive current density decreased after HPT processing, indicating a more protective oxide layer was formed on the surface of the HPT-processed alloy. Mott Schottky analysis confirmed the higher protection efficiency of the passive layer on the HPT-processed alloy. The film formed in the solution-treated alloy was a p-type and an n-type semiconductor while the alloy processed by HPT exhibited only an n-type semiconductor behavior. EIS showed that corrosion resistance in a saline medium increased with HPT processing.

Keywords: Aluminum alloy, corrosion, high-pressure torsion, ultrafine microstructure.

\section{Introduction}

Aluminum alloys are used extensively in civil construction and the automotive and aerospace industries due to their high strength-to-weight ratio, recyclability and high thermal and electrical conductivity. In addition, they have high corrosion resistance due to the development of protective oxide films on their surfaces when exposed at room temperature to environments containing oxygen ${ }^{1}$. Thus, these materials are more likely to undergo localized corrosion due to defects on the passive protective film. Localized corrosion is usually initiated at the oxide layer at sites weakened by chloride attacks, although chemical or physical heterogeneities at the surfaces may act as preferential nucleation sites ${ }^{2}$.

It is now well known that the mechanical properties of aluminum alloys significantly improve after processing by severe plastic deformation (SPD) ${ }^{3}$. The intense deformation leads to an increase in the dislocation density and the formation of low-angle boundaries that gradually evolve into high-angle boundaries promoting grain refinement throughout the bulk sample ${ }^{4}$. Because of the intense straining and grain refinement, these materials generally exhibit increased mechanical strength, good ductility and high resistance against fatigue and wear at low temperatures, as well as excellent superplastic properties at high temperatures ${ }^{5-9}$. Amongst SPD techniques, high-pressure torsion (HPT) $)^{10,11}$ permits the production of materials with finer grain structures and usually higher hardness through the application of high hydrostatic pressures together with torsional straining in disk-shaped samples.

The minimum grain size attained in Al alloys after HPT processing depends upon the amount of alloying elements together with the homologous temperature ${ }^{12}$. Accordingly, the addition of small amounts of $\mathrm{Mg}$ solutes in aluminum decreases its recovery rate during severe plastic deformation which ultimately generates finer grain structures than in the pure metal ${ }^{13,14}$. Conversely, SPD-processed metals recrystallize at lower temperatures than their counterparts produced through conventional metal-working procedures ${ }^{15}$ and dispersoid-forming elements such as $\mathrm{Sc}$ and $\mathrm{Zr}$ are added to $\mathrm{Al}-\mathrm{Mg}$ alloys to enhance their microstructural stability at high temperatures ${ }^{16,17}$. 
Although several investigations were carried out evaluating the corrosion behavior of $\mathrm{Al}$ alloys ${ }^{1,2}$, the effect of severe plastic deformation is not yet understood. It is generally accepted that localized corrosion, such as pitting and intergranular corrosion, decreases with a reduction in grain $\operatorname{size}^{18}$. However, the grain refinement introduced by severe plastic deformation is associated with a significant increase in other crystalline defects. Among the few investigations in this area, most used equal-channel angular pressing (ECAP) as the processing technique ${ }^{18}$. Accordingly, the objective of the present study was to examine and compare the corrosion behavior of $\mathrm{Al}-3 \% \mathrm{Mg}-0.2 \% \mathrm{Sc}$ (in wt $\%$ ) alloys before and after severe plastic deformation by high-pressure torsion.

\section{Experimental Procedures}

An $\mathrm{Al}-3 \% \mathrm{Mg}-0.2 \% \mathrm{Sc}$ (in wt.\%) alloy was used in this study with the material supplied by China Rare Metal Material Corporation (Jiangxi Province, China) in the form of forged bars having lengths of $130 \mathrm{~mm}$ and diameters of $10 \mathrm{~mm}$. These bars were solution treated at $880 \pm 2 \mathrm{~K}$ for $1 \mathrm{~h}$ and then quenched in cold water. After heat treatment, disks with a thickness of $\sim 1 \mathrm{~mm}$ were cut from the bars and then ground using abrasive papers to final thicknesses of $\sim 0.82 \mathrm{~mm}$. These disks were processed through 10 turns of HPT under quasi-constrained conditions ${ }^{19}$ at an initial temperature of $300 \mathrm{~K}$ using a rotation rate of $1 \mathrm{rpm}$ and a nominal pressure of $6.0 \mathrm{GPa}$.

The grain structure was determined using conventional metallographic procedures. Samples were mounted, ground and polished to a mirror-like quality using diamond paste and the final polishing was performed using colloidal silica solution $(0.06 \mu \mathrm{m})$. The samples were etched using a solution with $5 \%$ fluoroboric acid $\left(\mathrm{HBF}_{4}\right)$. The microstructure of the solution-treated alloy was examined at the mid-section of the samples using an Olympus BX51 optical microscope. The grain structure of the HPT-processed metal was observed at positions located at a distance of $\sim 3 \mathrm{~mm}$ from the center of the disk using a JSM6500F thermal field emission scanning electron microscope (SEM). The samples used in the corrosion tests were ground to \#2000 grit. After the corrosion tests, the samples were analyzed by SEM with energy dispersive X-ray spectrometry (SEM-EDX, Quanta FEG 3D FEI-Bruker X Flash 4.0).

The electrochemical tests were carried out in $3.5 \mathrm{wt} . / \mathrm{v} . \%$ $\mathrm{NaCl}$ aqueous solution ( $\mathrm{pH}$ of 7.3 and $4.49 \mathrm{mg} . \mathrm{L}^{-1}$ oxygen content) using an Autolab PGSTAT 100N potentiostat. An electrochemical cell with three electrodes was used having a working electrode and a counter electrode of platinum. The reference electrode was $\mathrm{Ag} / \mathrm{AgCl}$ and all potentials described hereafter refer to this electrode at $298 \mathrm{~K}$. The microstructure of the Al alloy was homogeneous along the working electrode (WE) and its exposed surface was 0.78 $\mathrm{cm}^{2}$. All tests were performed in triplicate. Cyclic polarization tests were performed at room temperature and the curves were obtained from the open circuit potential (OCP) at a fixed scan of $1 \mathrm{mV} / \mathrm{s}$. The corrosion potential $\left(\mathrm{E}_{\text {corr }}\right)$ was obtained as the stabilized open circuit potential. The corrosion current density, $i_{\text {corr }}$, was determined as the intersection between the corrosion potential with the straight line of Tafel region in the anodic branch. The scan direction was reversed when the current density reached $1 \mathrm{~mA} . \mathrm{cm}^{-2}$. The pitting potential was obtained by the tangent method.

Electrochemical impedance spectroscopy (EIS) tests were performed at a frequency range from $10 \mathrm{kHz}$ to 10 $\mathrm{mHz}$, with a potential amplitude of $20 \mathrm{mV}$ in relation to the corrosion potential. ZView software was used for data fitting of the impedance spectra and ZSimpWin software was used to validate the impedance data.

Mott-Schottky analysis was performed on the passive film at a frequency of $1 \mathrm{kHz}$ using a $10 \mathrm{mV}$ sinusoidal signal and steps of $20 \mathrm{mV}$ in the anodic direction. In order to evaluate the stability of the passive layer, a chronoamperometric measurement was carried out using an applied potential of $25 \mathrm{mV}$ below the $\mathrm{E}_{\text {pit }}$ with $7000 \mathrm{~s}$ of duration.

The surface layer was analyzed by X-ray photoelectron spectroscopy using a SPECS-Model Phoibus 100 equipment. The high-resolution spectra were obtained using a $\mathrm{K} \alpha$ radiation from an aluminum anode (photon energy: 1486.6 eV/line width: $0.85 \mathrm{eV}$ ). The $\mathrm{C} 1 \mathrm{~s}$ peak of adventitious carbon $(284.73 \mathrm{eV})$ was used as a reference for the spectra energy calibration. Peak fitting of high-resolution spectra was carried out using Casa XPS software.

\section{Results and Discussion}

\subsection{Microstructure}

Representative images of both the solution treated and the HPT-processed alloy are shown in Fig. 1 where the former was determined by optical microscopy and the latter by scanning electron microscopy. The average grain size in the solution-treated alloy was $\sim 300 \mu \mathrm{m}$ and HPT processing reduced the grain size by over 3 orders of magnitude to $\sim 140 \mathrm{~nm}$. It should be noted that the grain size after HPT processing agrees with previous reports for this alloy ${ }^{14,20}$ and the grain structures appeared to be homogeneously distributed throughout the entire areas of the SEM images.

\subsection{Cyclic polarization}

Representative curves of the OCP evolution for immersion times of 3600 seconds for both alloys are shown in Fig. 2. The alloy processed by HPT exhibited a more positive corrosion potential than the solution-treated alloy thereby suggesting a nobler behavior in this medium.

Figure 3 shows the characteristic cyclic polarization curves for the solution-treated and the HPT-processed alloys. Table 1 presents the values of the corrosion potential $\left(\mathrm{E}_{\text {corr }}\right)$, 


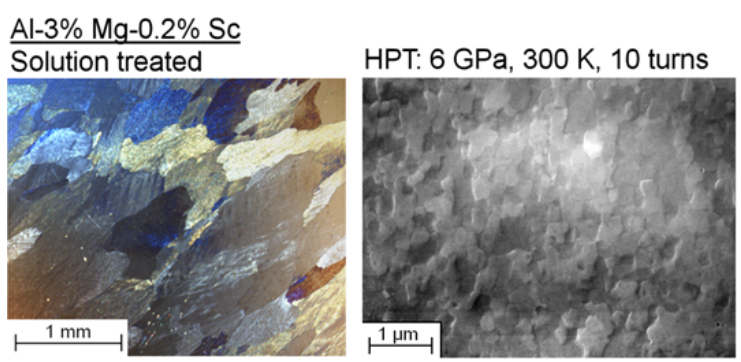

Figure 1. Grain structure of the $\mathrm{Al}-3 \% \mathrm{Mg}-0.2 \% \mathrm{Sc}$ alloy (a) after solution treatment at $880 \mathrm{~K}$ for $1 \mathrm{~h}$ (optical microscopy) and (b) after processing through 10 turns of HPT at room temperature (SEM).

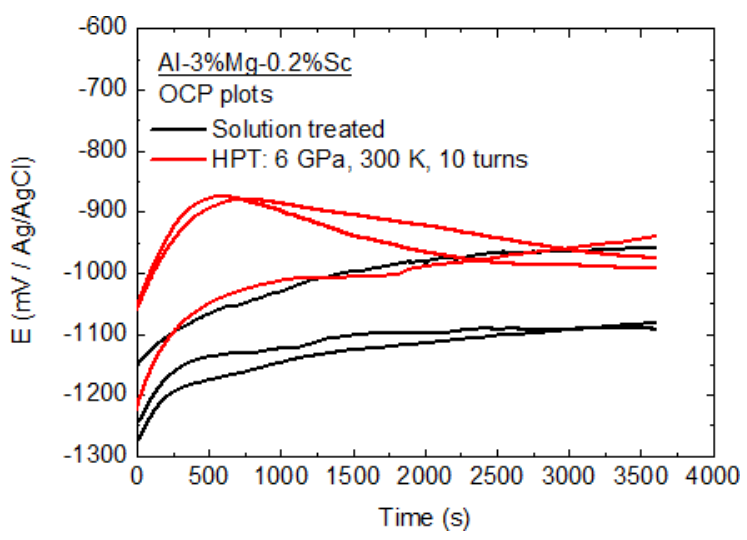

Figure 2. Open circuit potential variation with time for solution treated and HPT-processed alloy in $3.5 \mathrm{wt} . / \mathrm{v} . \% \mathrm{NaCl}$ solution

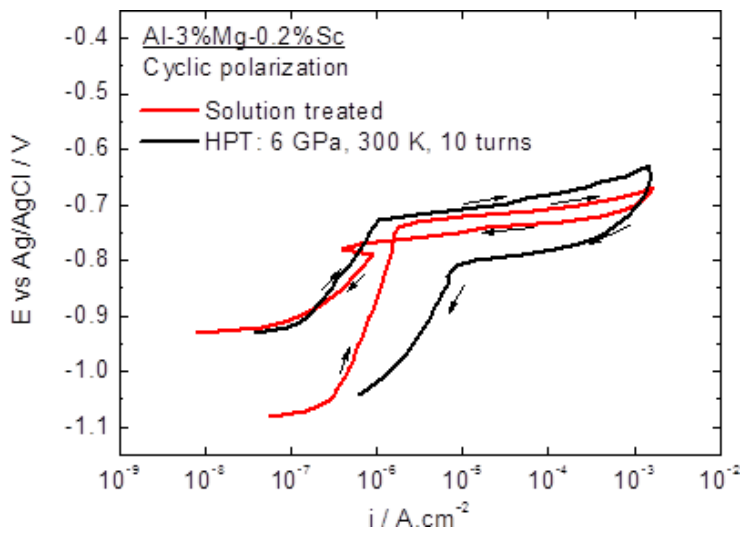

Figure 3. Cyclic polarization curves of solution treated and HPTprocessed alloy in $3.5 \mathrm{wt} . / \mathrm{v} . \% \mathrm{NaCl}$ solution

Table 1. Cyclic polarization results of the different samples in 3.5 wt./v. $\% \mathrm{NaCl}$ solution

\begin{tabular}{lcc}
\hline & Solution treated & HPT \\
\hline $\mathrm{E}_{\text {corr }}\left(\mathrm{mV}_{\mathrm{Ag} / \mathrm{AgCl}}\right)$ & $-1053.3 \pm 72.3$ & $-966.7 \pm 25.2$ \\
$\mathrm{i}_{\text {corr }}\left(\mu \mathrm{A} / \mathrm{cm}^{2}\right)$ & $11.3 \pm 1.2$ & $7.6 \pm 0.9$ \\
$\mathrm{E}_{\text {pit }}\left(\mathrm{mV}_{\mathrm{Ag} / \mathrm{AgCl}}\right)$ & $-735.3 \pm 11.8$ & $-727.1 \pm 10.8$ \\
$\mathrm{i}_{\text {pass }}\left(\mathrm{nA} / \mathrm{cm}^{2}\right)$ & $864.0 \pm 27.1$ & $399.0 \pm 63.8$ \\
$\mathrm{E}_{\text {prot }}\left(\mathrm{mV}_{\mathrm{Ag} / \mathrm{AgCl}}\right)$ & $-767.2 \pm 4.4$ & - \\
\hline
\end{tabular}

corrosion current density $\left(\mathrm{i}_{\text {corr }}\right)$, pitting potential $\left(\mathrm{E}_{\text {pit }}\right)$, passive current density $\left(\mathrm{i}_{\text {pass }}\right)$ and protection potential $\left(\mathrm{E}_{\text {prot }}\right)$.

The open circuit potential was measured before the electrochemical tests until stabilization. Polarization curves shown in Fig. 3 started at the corrosion potential. The corrosion current densities, $\mathrm{i}_{\text {corr }}$, of the solution treated alloy were slightly higher than the $i_{\text {corr }}$ after HPT, although the HPT sample showed a larger number of active sites such as grain boundaries and dislocations. The pitting potential (or breakdown potential) is clearly defined in Fig. 3 as the potential in which there is a rapid increase in current density. The passive current density was determined as the value in the middle of the passive potential range. Upon scan direction reversal, the current density decreases with decreasing potential until intersecting the forward curve in the passivation region. The protection potential, $\mathrm{E}_{\text {prot }}$, was determined as the electrode potential at this intersection where it is expected that the growth of active pits is diminished or possibly stopped.

To better understand the surface reactions in this Al alloy the surface features were characterized using SEM after the polarization corrosion tests. Figure 4 shows SEM micrographs for both $(\mathrm{a}, \mathrm{b})$ the solution treated and $(\mathrm{c}, \mathrm{d})$ the HPT-processed alloy after polarization tests. These images reveal deeper pits with a higher diameter on the surface of the solution treated alloy compared to the material processed by HPT.

The corrosion potential is $-966.7 \pm 25.2 \mathrm{mV}$ for HPTprocessed samples and the solution treated metal presented a lower corrosion potential of $-1053.3 \pm 72.3 \mathrm{mV}$; this value
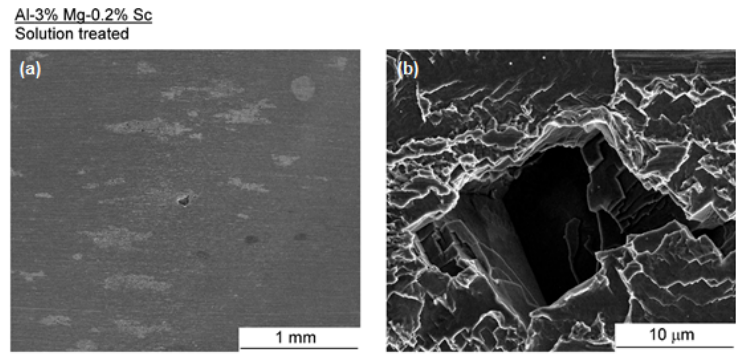

HPT: 6 GPa, R.T., 10 turns
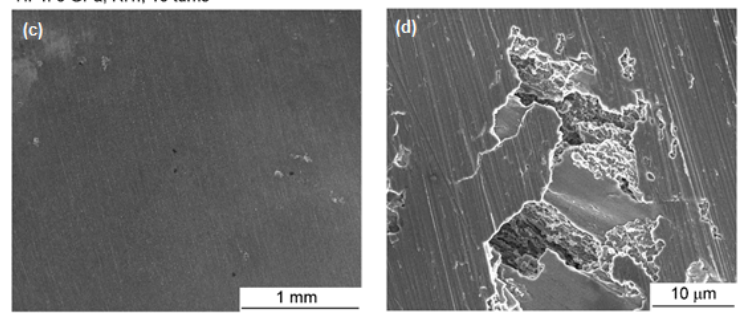

Figure 4. SEM micrographs of the surface of the $(a, b)$ solution treated and the $(\mathrm{c}, \mathrm{d})$ HPT-processed alloy, at $(\mathrm{a}, \mathrm{c})$ low and $(\mathrm{b}, \mathrm{d})$ high magnification, obtained after anodic polarization test in 3.5 wt./v.\% $\mathrm{NaCl}$ solution 
is consistent with an earlier report for an untreated $2024 \mathrm{Al}$ alloy $^{21}$. Both materials showed an initial increase in OCP which is attributed to the formation of corrosion products such as oxides and hydroxides.

The Al-3Mg-0.2Sc alloy shows a passivation behavior in both processing conditions and there was no difference between the pitting potential values for the two samples. On the other hand, the passive current density decreased from $885.5 \mathrm{nA} / \mathrm{cm}^{2}$ for the solution treated alloy to 393.2 $\mathrm{nA} / \mathrm{cm}^{2}$ after HPT processing. This reduction is attributed to the finer grain structure and higher dislocation density in the latter which promotes the formation of a more compact and protective corrosion product. Nevertheless, these defects are preferred sites for nucleation of pits. As a consequence, both samples display similar values of pitting potential.

These results suggest that the intense straining imposed by HPT can effectively increase the corrosion resistance of the aluminum alloy, thereby increasing the protection efficiency of the passive layer. The solution-treated and HPT-processed alloys have positive hysteresis loops indicating the possibility of a localized corrosion ${ }^{22}$. A repassivation of the solution-treated alloy was identified and the protection potential was measured as $-765.3 \mathrm{mV}$. By contrast, the HPTprocessed alloy showed no repassivation. The propagation of pits is expected to affect the protection potential $E_{\text {prot. }}$ As the corrosion pits on the surface of the solution treated samples were larger and deeper (Fig. 4) in comparison with the HPT-processed alloy, the pit propagation was greater. This effect increases the amount of corrosion products which could facilitate the repassivation.

\subsection{Electrochemical impedance spectroscopy analysis}

The Nyquist plots are presented in Fig. 5. Both samples showed a corrosion mechanism with two time-constants. It appears that the HPT-processed alloy showed a diffusion control of the corrosive process, and this hypothesis was confirmed by data fitting. The equivalent circuits used to describe the corrosion behavior of the Al-3Mg-0.2Sc alloy were $\mathrm{R}_{\mathrm{s}}\left(\mathrm{CPE}_{\mathrm{ox}} \mathrm{R}_{\mathrm{ox}}\right)\left(\mathrm{CPE}_{\mathrm{dl}} \mathrm{R}_{\mathrm{ct}}\right)$ for the solution treated and $\mathrm{R}_{\mathrm{s}}\left(\mathrm{CPE}_{\mathrm{ox}} \mathrm{R}_{\mathrm{ox}}\right)\left(\mathrm{CPE}_{\mathrm{dl}} \mathrm{R}_{\mathrm{ct}} \mathrm{W}\right)$ for the HPT-processed alloy. In the equivalent circuit of solution treated alloy and HPT alloy, the resistance of the electrolyte, $R_{s}$, is placed in series with the constant phase element and impedance of the oxide layer, $\mathrm{CPE}_{\text {ox }}$ and $\mathrm{R}_{\mathrm{ox}}$, respectively. Also, the capacitance of the double layer (represented by the constant phase element) and the charge transfer resistance, $\mathrm{CPE}_{\mathrm{dl}}, \mathrm{R}_{\mathrm{ct}}$, are placed in series with $\mathrm{CPE}_{\text {ox }}$ and $\mathrm{R}_{\mathrm{ox}}$. This circuit is consistent with other reports in the literature ${ }^{2,23,24}$ for $\mathrm{Al}$ alloys. The impedance spectra were fitted to this circuit for both alloys with a very good correlation with $\chi^{2}$ on the order of $10^{-4}$ (Table 2 ). The values of the fitting parameters are given in Table 2. As expected, the value of Rs is similar in both conditions since

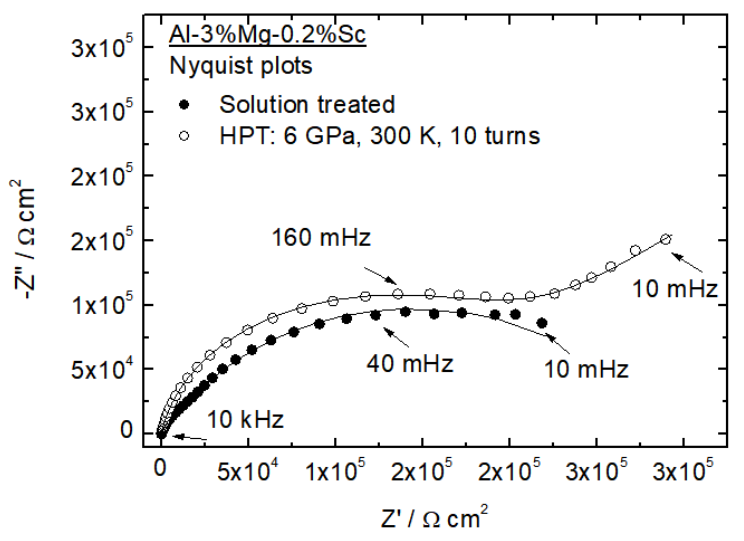

Figure 5. Nyquist diagrams of the solution treated and the HPTprocessed alloys in $3.5 \mathrm{wt} . / \mathrm{v} . \% \mathrm{NaCl}$ solution after $1 \mathrm{~h}$ stabilization at the OCP

Table 2. Fitting results of electrochemical parameters for solution treated and HPT processed alloys.

\begin{tabular}{lcc}
\hline & Solution treated & HPT \\
\hline $\mathrm{Rs}\left(\Omega \cdot \mathrm{cm}^{2}\right)$ & 23.19 & 23.02 \\
$\mathrm{CPE}_{\mathrm{ox}}\left(\Omega^{-1} \cdot \mathrm{s}^{\mathrm{n}} \cdot \mathrm{cm}^{-2}\right)$ & $1.49 \times 10^{-5}$ & $4.55 \times 10^{-6}$ \\
$\mathrm{n}_{1}$ & 0.91 & 0.91 \\
$\mathrm{R}_{\mathrm{ox}}\left(\Omega \cdot \mathrm{cm}^{2}\right)$ & $1.35 \times 10^{4}$ & $1.88 \times 10^{5}$ \\
$\mathrm{CPE}\left(\Omega^{-1} \cdot \mathrm{s}^{\mathrm{n}} \cdot \mathrm{cm}^{-2}\right)$ & $1.48 \times 10^{-5}$ & $2.55 \times 10^{-5}$ \\
$\mathrm{n}_{2}$ & 0.86 & 0.88 \\
$\mathrm{R}_{\mathrm{ct}}\left(\Omega \cdot \mathrm{cm}^{2}\right)$ & $2.34 \times 10^{5}$ & $2.19 \times 10^{7}$ \\
$\mathrm{~W}\left(\Omega \cdot \mathrm{cm}^{2}\right)$ & - & $2.39 \times 10^{6}$ \\
$\chi^{2}$ & $5.01 \times 10^{-4}$ & $2.06 \times 10^{-4}$ \\
\hline
\end{tabular}

this parameter is associated with the electrolyte resistance. In practice, this value depends on the distance between the working and the reference electrode together with the concentration of the solution.

The $\mathrm{CPE}_{\text {ox }}$ values are related to the high frequency range and are associated with the oxide layer exposed to the corrosive medium. Oxide capacitance is attributed to the ion transport through the oxide layer ${ }^{23}$. The impedance of the passive layer $\left(\mathrm{R}_{\mathrm{ox}}\right)$ of the HPT-processed alloy is one order of magnitude higher than the oxide layer on the surface of the solution-treated Al alloy. This is consistent with the lower passive current density observed for the ultrafine-grained alloy. At high frequencies, the current mainly passes through the oxide film. An increase in the $\mathrm{R}_{\mathrm{ox}}$ value can be related to a reduction in the corroded area ${ }^{24}$. The HPT-processed alloy showed a higher value of $\mathrm{R}_{\mathrm{ct}}$ than the solution-treated alloy, increasing the inhibition for the charge transfer at the metal/oxide interface ${ }^{2}$. Therefore, the Al alloy processed by HPT showed a higher corrosion resistance than the solutiontreated alloy based on the EIS results, mainly on the higher values of oxide impedance and $\mathrm{R}_{\mathrm{ct}}$. 
Bode plots are presented in Fig. 6. The curves clearly display two maxima in the phase angle versus frequency diagram for the solution-treated alloy and a broad maximum in the phase angle plot for the HPT-processed alloy, thus confirming the presence of two time-constants. A time constant at high frequencies may indicate the presence of an oxide film on the metal matrix. In addition, a second time constant at lower frequencies can indicate the occurrence of corrosion processes occurring at the metal/oxide interface ${ }^{25}$. Analyzing the Bode diagrams, the $\mathrm{Al}$ alloy processed by HPT showed a higher impedance modulus than the solution-treated alloy which agrees with the Nyquist diagram results.

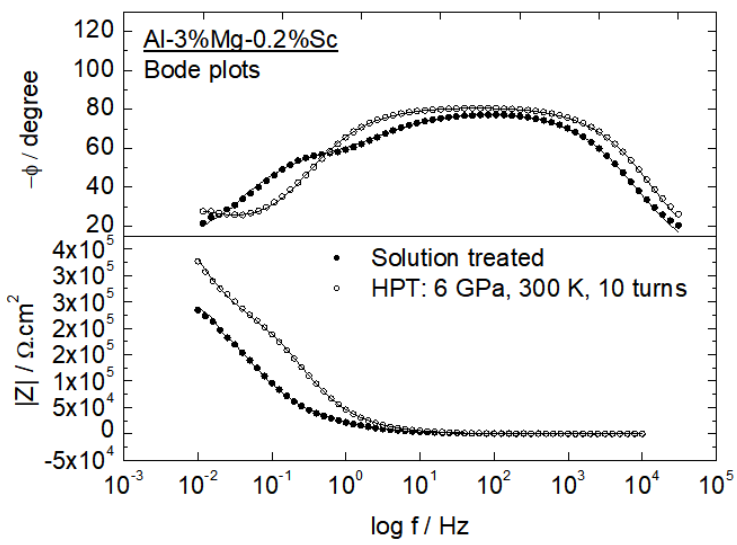

Figure 6. Bode diagrams of impedance modulus and phase angle versus frequency of solution treated and HPT-processed alloy in 3.5 wt. $\% \mathrm{NaCl}$ solution

\subsection{Mott-Schottky analysis}

The Mott-Schottky analysis is a common method for evaluating the behavior of passive films ${ }^{26}$. The density of defects that act as charge donors or acceptors are estimated from the following equations 1 and 2 respectively:

$$
\begin{aligned}
& \frac{1}{C_{S C}^{2}}=\frac{2}{e \varepsilon \varepsilon_{0} N_{d}}\left(E-E_{f b}-\frac{k T}{e}\right) \\
& \frac{1}{C_{S C}^{2}}=\frac{-2}{e \varepsilon \varepsilon_{0} N_{a}}\left(E-E_{f b}-\frac{k T}{e}\right)
\end{aligned}
$$

In this analysis $C_{s c}$ is the space charge capacitance, $\varepsilon$ is the electron charge $\left(1.6 \times 10^{-19} \mathrm{C}\right), \varepsilon$ is the dielectric constant of the $\mathrm{Al}$ oxide (which is considered as 10 ) ) $^{22}, \varepsilon_{0}$ is the vacuum permittivity $\left(8.85 \times 10^{-14} \mathrm{~F} \mathrm{~cm}^{-1}\right), \mathrm{N}_{\mathrm{d}}$ is the donor density in n-type semiconductors and $\mathrm{N}_{\mathrm{a}}$ is the acceptor density in p-type semiconductors, $\mathrm{E}$ is the applied potential, $\mathrm{E}_{\mathrm{fb}}$ is the flat-band potential, $\mathrm{k}$ is Boltzmann's constant $\left(1.38 \times 10^{-23}\right.$ $\mathrm{J} . \mathrm{K}^{-1}$ ) and $\mathrm{T}$ is the absolute temperature. It follows that the densities of donors and acceptors can be estimated from the inclination of plots of $1 / C_{s c}{ }^{2}$ as a function of $E$ in the passivation range.

The measurement was performed from the initial potential of $-1.06 \mathrm{~V}$ to the final potential of $-0.74 \mathrm{~V}$ for the solution treated alloy and from an initial potential of $-0.92 \mathrm{~V}$ to the final potential of $-0.73 \mathrm{~V}$ for the alloy processed by HPT. Such plots are given in Fig. 7 for the solution treated and HPT-processed alloys. The curves show a positive slope for the HPT-processed material indicating an n-type semiconductor behavior. The solution treated material exhibits an initial positive slope (n-type behavior) but also a negative slope characteristic of p-type behavior above $-0.95 \mathrm{~V}_{\mathrm{Ag} / \mathrm{AgCl}}$. The densities of carriers (donors) were calculated as $2.65 \times$ $10^{20} \mathrm{~cm}^{-3}$ and $0.94 \times 10^{20} \mathrm{~cm}^{-3}$ for the solution treated and HPT-processed material, respectively. The lower density of donors of the passive film of the HPT-processed alloy than for the solution-treated alloy agrees with the highest oxide impedance observed in the EIS results and the lowest passive current density identified in the cyclic polarization results. A reduction in carrier density has been reported in a magnesium alloy AZ91 and attributed to grain refinement ${ }^{27}$. This observation also supports the hypotheses that a higher concentration of grain boundaries and dislocations on the surface of the HPT-processed alloy, which are anodic sites in the corrosion process, contributes to generate a more compact, homogeneous and protective oxide layer.

The oxidized surfaces of the different samples were analyzed by X-ray Photoelectron Spectroscopy (XPS) and no significant differences were observed between them. Figure 8 shows the high-resolution spectra in the oxygen energy range (O1s) for (a) the solution treated and (b) the HPT-processed alloy. The results revealed the presence of a peak at the binding energy interval of $\sim 528-535 \mathrm{eV}$. The peak was adjusted using two Gaussians to provide more detailed information concerning the bonds at the surface of the $\mathrm{Al}$ samples. For both processing conditions, one peak indicates the bonding with aluminum forming $\mathrm{Al}_{2} \mathrm{O}_{3}$ and the remaining peak is associated with $\mathrm{Mg}-\mathrm{Al}-\mathrm{O}$ bonds likely from the $\mathrm{MgAl}_{2} \mathrm{O}_{4}$ phase. The high-resolution spectra in the aluminum energy range (Al2s) have confirmed the presence of $\mathrm{MgAl}_{2} \mathrm{O}_{4}$. It is estimated that most of the sample surface is formed by the $\mathrm{MgAl}_{2} \mathrm{O}_{4}$ phase while the $\mathrm{Al}_{2} \mathrm{O}_{3}$ phase occupies a smaller but important portion.

Magnesium and aluminum oxides are usually n-type semiconductors. This agrees with the behavior of the oxides in the surface of the HPT-processed alloy. The solution treated alloy exhibits an n-type that changes to p-type above -0.95 $\mathrm{VAg} / \mathrm{AgCl}$. It is worth noting that a change from n-type to p-type semiconductor has been reported during corrosion of aluminum and attributed to the presence of chloride ions in the solution ${ }^{28}$. Further research is needed to clarify the reasons such a transition takes place in the solution treated but not in the HPT-processed alloy. 

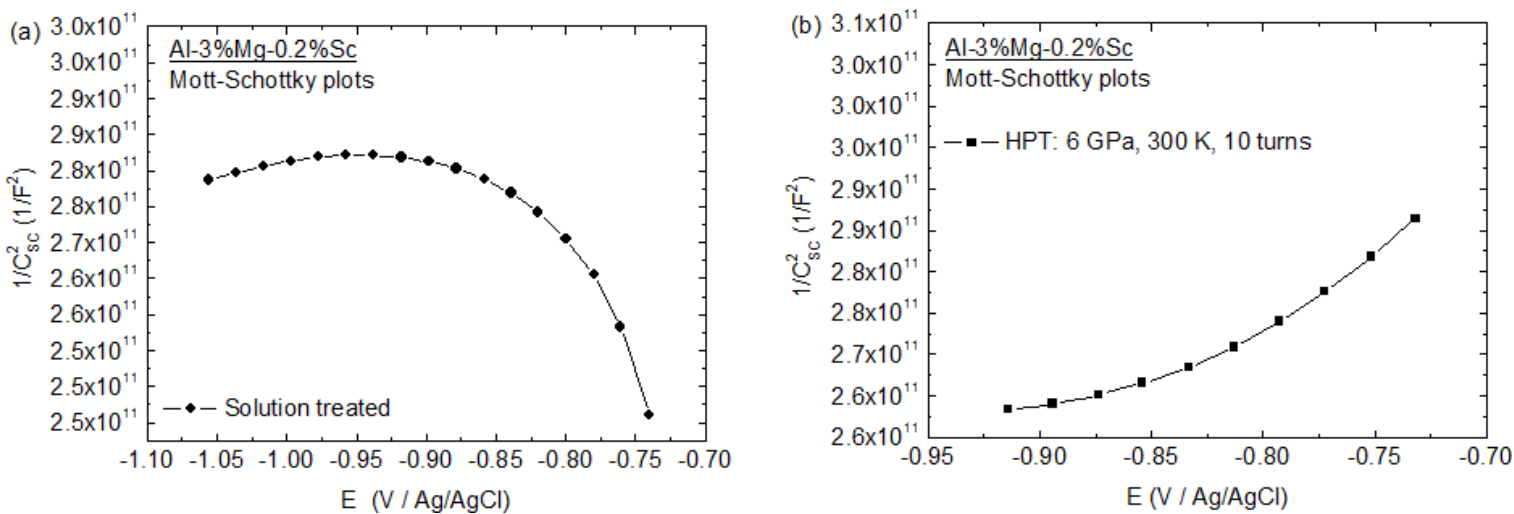

Figure 7. Mott-Schottky plots for (a) the solution treated and (b) the HPT-processed alloy in $3.5 \mathrm{wt} . / \mathrm{v}$. $\mathrm{NaCl}$ solution at $1000 \mathrm{~Hz}$
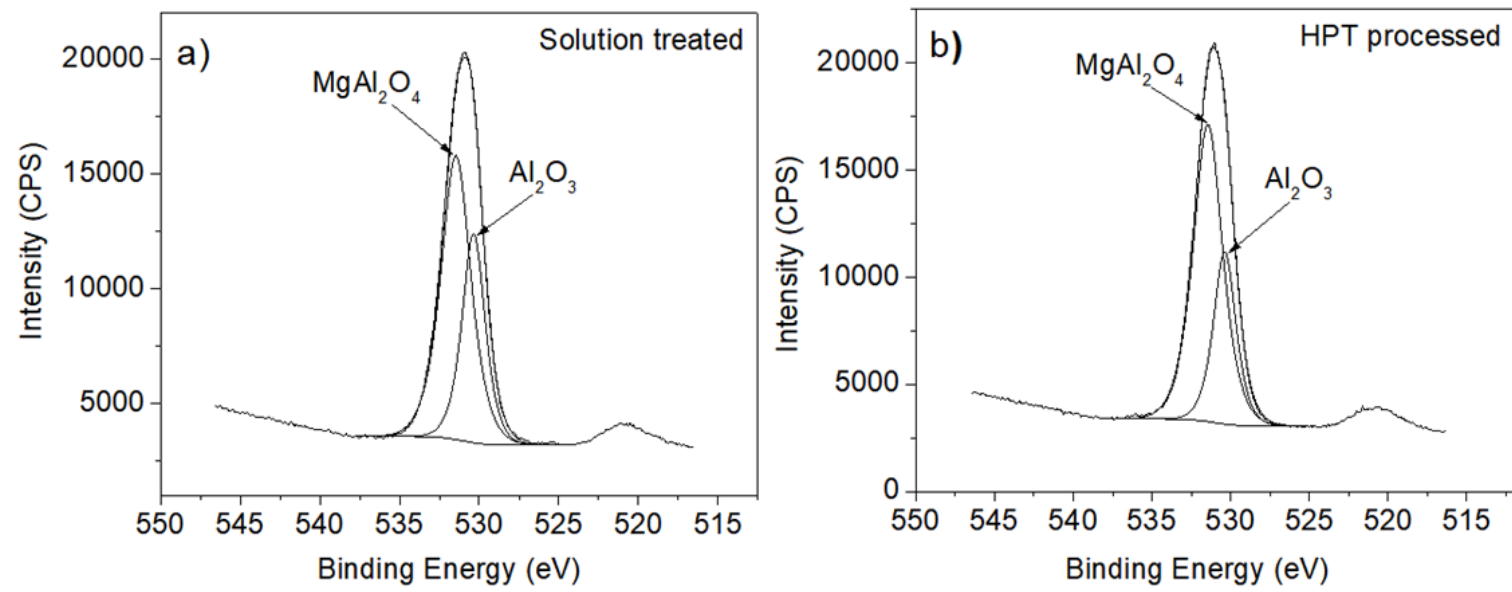

Figure 8. The high resolution spectrum in the oxygen energy range (O1s) for (a) the solution treated and (b) the HPT-processed alloy.

\subsection{Chronoamperometric measurements}

To evaluate the oxide film behavior of the alloys immediately before the film degradation, a potential of $25 \mathrm{mV}$ was applied below the $\mathrm{E}_{\text {pit }}$ for both alloys. Typical current signatures recorded during the chronoamperometric measurements are shown in Fig. 9. The current decreased with time for the solution-treated alloy and this was mainly due to the formation and thickening of the passive film which shows a dissolution rate which is negligible since the applied potential was in the passivation range ${ }^{29,30}$. In this case, the passive current density is stable. For the alloy processed by HPT, the current density grows with time, probably due to the initiation of pitting corrosion or nucleation of metastable pits ${ }^{6}$. It is also noted that the current density of the alloy processed by HPT is considerably higher than for the solution-treated alloy, indicating that the passive film on the HPT-processed alloy initiated its degradation at this potential.

Transients in current could be observed for both alloys. The small fluctuations in current density in Fig. 9 suggest the occurrence of metastable pits which were nucleated and then repassivated ${ }^{6}$. At the end of the passive region, where the chronoamperometry measurements were recorded, there

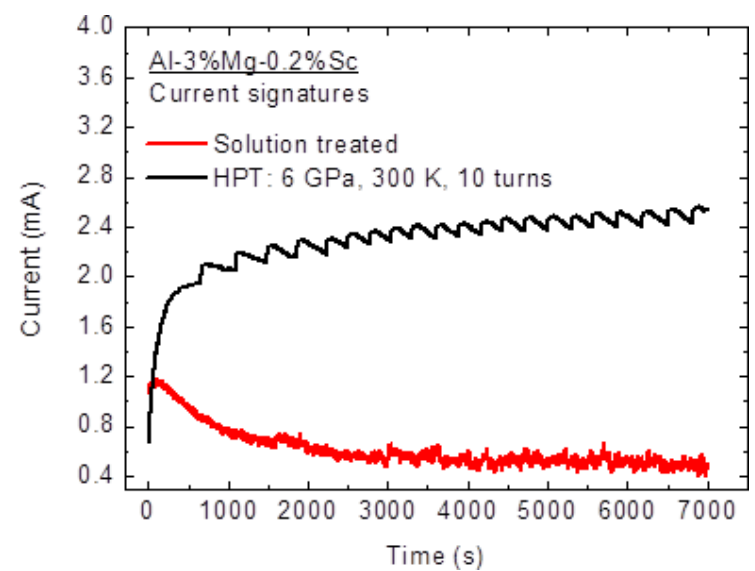

Figure 9. Current signatures recorded during chronoamperometric measurements of solution treated and HPT-processed alloys in 3.5 wt./v. $\mathrm{NaCl}$ solution

is a higher occurrence of metastable pits that have a greater tendency to repassivate in the case of the solution treated alloy. Thus, the current obtained for the solution treated alloy was lower than the alloy processed by HPT. The result agrees with the observation that the solution treated alloy presented a better ability to repassivate than the HPT-processed alloy. 
Both currents are larger than the passive current densities indicating the degradation of the passive layers for the two alloys at this potential.

\subsection{Final considerations}

The HPT-processed alloy showed a higher impedance and a lower passive current density than the solution treated alloy in a saline solution. This result agrees with the n-type semiconductor behavior of $\mathrm{Mg}$ and $\mathrm{Al}$ oxides found on the surface of the HPT-processed alloy. The solution treated alloy showed a bi-layer film on its surface, with an n-p junction, which is less protective than a pure n-type semiconductor found on the surface of the ultrafine-grained alloy. The beneficial aspect of the solution treated alloy was its repassivation ability. This alloy showed larger pits with a higher diameter and a greater pit propagation that produced a repassivation in the saline medium.

\section{Conclusions}

1. This report compares the corrosion behavior of an $\mathrm{Al}-3 \% \mathrm{Mg}-0.2 \% \mathrm{Sc}$ alloy after solid solution treatment and after HPT processing. The latter exhibits a grain size $\sim 3$ orders of magnitude smaller than in the unprocessed alloy.

2. HPT processing leads to a more protective passive layer with a lower passive current density evaluated by using cyclic polarization tests, a lower number of donors in the oxide film (an n-p semiconductor) analyzed using a Mott Schottky technique, and a higher impedance of oxide layer evaluated by EIS data.

3. A SEM analysis of the corroded surface revealed broader and deeper pits on the surface of the solution-treated alloy than on the surface of the HPT-processed alloy.

4. The solution treated alloy showed a repassivation behavior in an aqueous solution of $3.5 \mathrm{wt} . / \mathrm{v} . \% \mathrm{NaCl}$.

5. The solution treated alloy showed a bi-layer film with a p-n junction of the oxide layer in an aqueous solution of 3.5 wt./v.\% NaCl.

\section{Acknowledgements}

The authors acknowledge support from Centro de Microscopia da Universidade Federal de Minas Gerais, CAPES, CNPq and FAPEMIG.

\section{References}

1. Melchers RE. Bi-modal trend in the long-term corrosion of aluminum alloys. Corrosion Science. 2014;82:239-247.
2. Moreto JA, Marino CEB, Bose Filho WW, Rocha LA, Fernandes JCS. SVET, SKP and EIS study of the corrosion behavior of high strength $\mathrm{Al}$ and $\mathrm{Al}-\mathrm{Li}$ alloys used in aircraft fabrication. Corrosion Science. 2014;84:30-41.

3. Sabirov I, Murashkin MY, Valiev RZ. Nanostructured aluminum alloys produced by severe plastic deformation: new horizons in development. Materials Science and Engineering A: Structural Materials: Properties, Microstructure and Processing. 2013;560:124.

4. Valiev RZ, Estrin Y, Horita Z, Langdon TG, Zehetbauer MJ, Zhu YT. Producing Bulk Ultrafine-Grained Materials by Severe Plastic Deformation. JOM. 2006;58(4):33-39.

5. Ortiz-Cuellar E, Hernandez-Rodriguez MAL, García-Sanchez E. Evaluation of the tribological properties of an Al-Mg-Si alloy processed by severe plastic deformation. Wear. 2011;271(910):1828-1832.

6. Son IJ, Nakano H, Oue S, Kobayashi S, Fukushima H, Horita Z. Pitting corrosion resistance of anodized aluminum-cooper alloy processed by severe plastic deformation. Materials Transactions. 2008;49(11):2648-2655.

7. Ralston KD, Fabijanic D, Birbilis N. Effect of grain size on corrosion of high purity aluminum. Electrochimica Acta. 2011;56:1729-1736.

8. Kawasaki M, Langdon TG. Review: achieving superplastic properties in ultrafine-grained materials at high temperatures. Journal of Materials Science. 2016;51(1):19-32.

9. Pereira PHR, Huang Y, Kawasaki M, Langdon TG. An examination of the superplastic characteristics of Al-Mg-Sc alloys after processing. Journal of Materials Research. 2017;32(24):4541-4553.

10. Zhilyaev AP, Langdon TG. Using high-pressure torsion for metal processing: Fundamentals and applications. Progress in Materials Science. 2008;53(6):893-879.

11. Silva CLP, Oliveira AC, Costa CGF, Figueiredo RB, Leite MF, Pereira MM, et al. Effect of severe plastic deformation on the biocompatibility and corrosion rate of pure magnesium. Journal of Materials Science. 2017;52(10):5992-6003.

12. Edalati K, Akama D, Nishio A, Lee S, Yonenaga Y, Cubero-Sesin $\mathrm{JM}$, et al. Influence of dislocation-solute atom interactions and stacking fault energy on grain size of single-phase alloys after severe plastic deformation using high-pressure torsion. Acta Materialia. 2014;69:68-77.

13. Iwahashi Y, Horita Z, Nemoto M, Langdon TG. Factors influencing the equilibrium grain size in equal-channel angular pressing: role of $\mathrm{Mg}$ additions to aluminum. Metallurgical and Materials Transactions: A. 1998;29(10):2503-2510.

14. Pereira PHR, Huang Y, Langdon TG. Thermal stability and superplastic behaviour of an $\mathrm{Al}-\mathrm{Mg}-\mathrm{Sc}$ alloy processed by ECAP and HPT at different temperatures. IOP Conference Series: Materials Science and Engineering. 2017;194(1):1-6.

15. Wang J, Iwahashi Y, Horita Z, Furukawa M, Nemoto M, Valiev RZ, et al. An investigation of microstructural stability in an Al-Mg alloy with submicrometer grain size. Acta Materialia. 1996;44(7):2973-2982. 
16. Pereira PHR, Wang YC, Huang Y, Langdon TG. Influence of grain size on the flow properties of an Al-Mg-Sc alloy over seven orders of magnitude of strain rate. Materials Science and Engineering: A. 2017;685:367-376.

17. Avtokratova E, Sitdikov O, Mukhametdinova O, Markushev M, Murty SVSN, Prasad MJNV, et al. Microstructural evolution in $\mathrm{Al}-\mathrm{Mg}-\mathrm{Sc}-\mathrm{Zr}$ alloy during severe plastic deformation and annealing. Journal of Alloys and Compounds: C. 2016;673:182194.

18. Miyamoto H. Corrosion of ultrafine grained materials by severe plastic deformation, an overview. Materials Transactions. 2016;57(5):559-572.

19. Figueiredo RB, Pereira PHR, Aguilar MTP, Cetlin PR, Langdon TG. Using finite element modeling to examine the temperature distribution in quasi-constrained high-pressure torsion. Acta Materialia. 2012;60(6-7):3190-3198.

20. Sakai Z, Horita Z, Langdon TG. Grain refinement and superplasticity in an aluminum alloy processed by high-pressure torsion. Materials Science and Engineering: A. 2005;393(1-2):344-351.

21. Yin B, Fang L, Tang AQ, Huang QL, Hu J, Mao JH, et al. Novel strategy in increasing stability and corrosion resistance for super-hydrophobic coating on aluminum alloy surfaces. Applied Surface Science. 2011;258:580-585.

22. Liu Y, Meng GZ, Cheng YF. Electronic structure and pitting behavior of 3003 aluminum alloy passivated under various conditions. Electrochimica Acta. 2009;54(17):4155-4163.
23. Li L, He J, Lei J, Xu W, Jing X, Ou X, et al. A sol-bath-gel approach to prepare hybrid coating for corrosion protection of aluminum alloy. Surface and Coatings Technology. 2015;279:7278 .

24. Xiao YP, Pan QL, Li WB, Liu XY, He YB. Influence of heat treatment on corrosion behavior of Al-Zn-Mg-Cu-Zr-Sc alloy. Materials and Corrosion. 2012;63(5):421-430.

25. Mansfeld F, Lin S, Kim S, Shih H. Pitting and passivation of $\mathrm{Al}$ alloys and $\mathrm{Al}$ based metal matrix composites. Journal of Electrochemical Society. 1990;137(1):78-82.

26. Pech-Canul MA, Pech-Canul MI, Bartolo-Pérez P, Echeverría $\mathrm{M}$. The role of silicon alloying addition on the pitting corrosion resistance of an Al-12wt.\%Si alloy. Electrochimica Acta. 2014; 140:258-265.

27. Li Y, Zhang T, Wang F. Effect of microcrystallization on corrosion resistance of AZ91D alloy. Electrochimica Acta. 2006;51(14):2845-2850.

28. Zhang B, Li Y, Wang F. Observations of anomalies in the anodic behavior of microcrystalline aluminum. Corrosion Science. 2010;52(8):2612-2615.

29. Tian W, Li S, Wang B, Liu J, Yu M. Pitting corrosion of naturally aged AA 7075 aluminum alloys with bimodal grain size. Corrosion Science. 2016;113:1-16.

30. Narayanan R, Seshadri SK. Point defect model and corrosion of anodic oxide coatings on Ti-6Al-4V. Corrosion Science. 2008;50(6):1521-1529. 